\title{
Influences of material processing on the microstructure and inter- granular current properties of polycrystalline bulk $\mathrm{Ba}(\mathrm{Fe}, \mathrm{Co})_{2} \mathrm{As}_{2}$
}

\author{
Y. Hayashi ${ }^{\mathrm{a}}$, A. Yamamoto ${ }^{\mathrm{a}, \mathrm{b}, *}$, H. Ogino ${ }^{\mathrm{a}}$, J. Shimoyama ${ }^{\mathrm{a}}$, K. Kishio ${ }^{\mathrm{a}}$ \\ ${ }^{a}$ Department of Applied Chemistry, The University of Tokyo, 7-3-1 Hongo, Bunkyo-ku, Tokyo 113-8656, Japan \\ ${ }^{b}$ JST-PRESTO, 4-1-8 Honcho Kawaguchi, Saitama 332-0012, Japan
}

\begin{abstract}
The phase formation, microstructure, magnetic, and transport properties of $\mathrm{Ba}(\mathrm{Fe}, \mathrm{Co})_{2} \mathrm{As}_{2}$ polycrystalline bulks prepared by systematically varied synthesis conditions were studied to clarify the key issues for intergranular critical current properties. After optimization of heat treatment process, $\mathrm{Ba}\left(\mathrm{Fe}_{0.92} \mathrm{Co}_{0.08}\right)_{2} \mathrm{As}_{2}$ samples with high phase purity and $T_{\mathrm{c}}>25 \mathrm{~K}$ were reproducibly obtained. Electron microscopy analyses showed that the use of refined starting powder by high-energy ball-milling yield microstructure with improved uniformity and $\mathrm{Ba}(\mathrm{Fe}, \mathrm{Co})_{2} \mathrm{As}_{2}$ phase can be synthesized at lower temperature down to $500^{\circ} \mathrm{C}$ owing to an increased reactivity. The samples synthesized at low temperature showed well-connected microstructure with fine grain size and intergranular critical current density progressively improved with lowering the heating temperature. Our results suggest that fine grain size and grain boundary structure formed at low temperature are favorable for increasing the area of effective transport current path, while they are electromagnetically weakly coupled and suppressed under external magnetic field.
\end{abstract}

(C) 2014 The Authors. Published by Elsevier B.V.

Selection and peer-review under responsibility of the ISS 2013 Program Committee.

Keywords: (Iron-based superconductors; polycrystalline bulks; microstructure; intergranular current)

\section{Introduction}

The recent discovery of Fe-based superconductors (FBS) by Hosono et al. [1] opened a new possibility of highfield applications. Fe-based superconductors are belonging to different families (called in the following "1111" for

\footnotetext{
* Corresponding author. Tel.: +81-3-5841-7713; fax: +81-3-5841-7713.

E-mail address: yamamoto@appchem.t.u-tokyo.ac.jp
}

1875-3892 (C) 2014 The Authors. Published by Elsevier B.V.

Selection and peer-review under responsibility of the ISS 2013 Program Committee. 
REFeAsO, "122" for $\mathrm{AEFe}_{2} \mathrm{As}_{2}$ [2,3], and "11" for $\mathrm{Fe}(\mathrm{Te}, \mathrm{Se})$ [4] (RE denotes rare earth and $\mathrm{AE}$ denotes alkali earth)) with critical temperatures $\left(T_{\mathrm{c}}\right.$ ), in the optimally doped compounds, up to $55 \mathrm{~K}$ for 1111 and very high upper critical fields $\left(H_{\mathrm{c} 2}\right)$ approaching $\sim 300 \mathrm{~T}$ for 1111 [5] and $\sim 100 \mathrm{~T}$ for 122 doped with potassium [6] or cobalt [7]. Moreover, both single crystals and thin films show high intragranular critical current density $\left(J_{\mathrm{c}}^{\text {local }}\right)$ well above $10^{6}$ $\mathrm{A} / \mathrm{cm}^{2}$ owing to strong vortex pinning [8-14]. The electromagnetic anisotropy $\left(\gamma_{\mathrm{H}}\right)$ of FBSs is rather small, $\sim 5$ for $1111,1-2$ for 122 and 1-2 for 11, which is favorable for practical applications [15].

The early studies have shown that investigation of the critical current density $\left(J_{c}\right)$ of 1111 polycrystalline samples emphasized the strong electromagnetic granularity of these compounds[16-18]. Intergranular critical current density of a sample $\left(J_{c}{ }^{\text {global }}\right)$ in polycrystalline FBS materials is found to be largely reduced by intrinsic weak-link nature $[19,20]$ analogous to cuprates and/or structural defects at grain boundaries[21,22]. The intrinsic weak-link behavior was more explicitly shown by studies using cobalt doped Ba122 epitaxial films on bicrystal substrate with various misorientation angles $\left(\theta_{\mathrm{GB}}\right)[19,20]$. Katase et al. reported that the intergranular current density through bicrystal grain boundary remained high $\left(>1 \times 10^{6} \mathrm{~A} / \mathrm{cm}^{2}\right)$ and nearly constant up to $\theta_{\mathrm{GB}} \sim 9^{\circ}$, which is much larger than $\sim 5^{\circ}$ of YBCO [20]. Indeed, recently Weiss et al. reported high $J_{\mathrm{c}}^{\text {global }}$ in potassium or cobalt doped Ba122 wires [23]. More recent studies have shown that polycrystalline forms of 122 phase FBS wires with $J_{\mathrm{c}}{ }^{\text {global }} \sim 10^{4}-10^{5} \mathrm{~A} / \mathrm{cm}^{2}$ can be reproducibly fabricated $[24,25]$.

On the other hand, the reported values of $J_{\mathrm{c}}^{\text {global }}$ in FBS materials vary more than one order of magnitude among different systems and dopants, i.e. $\sim 10^{4}, \sim 10^{5}$ and $\sim 10^{3} \mathrm{~A} / \mathrm{cm}^{2}$ for 1111,122 and 11 [23-28], respectively, meaning that the limiting mechanism of intergranular current is unrevealed for general FBS polycrystalline materials. In addition, polycrystalline FBS samples are likely to form multi-phase since 1111 and 122 are quaternary or even quinary systems including chemically reactive and volatile elements, such as fluorine and potassium. To clarify the key factors affecting $J_{\mathrm{c}}{ }^{\text {global }}$ in FBSs, studies of grain boundaries and current transport properties using single-phase polycrystalline samples with well-defined intragranular superconducting properties would be greatly helpful.

In the present study, we have evaluated the microstructure and electromagnetic properties of high purity $\mathrm{Ba}(\mathrm{Fe}, \mathrm{Co})_{2} \mathrm{As}_{2}$ polycrystalline bulks synthesized by various techniques to establish the reproducible synthesis route of single-phase FBSs and to understand the effective ways for achieving high $J_{\mathrm{c}}^{\text {global }}$. Among various FBS compounds, $\mathrm{Ba}(\mathrm{Fe}, \mathrm{Co})_{2} \mathrm{As}_{2}[3]$ was chosen for this study, since its carrier doping element, cobalt, is chemically stable and thus can be precisely controllable during multiple heat treatments and powder processing. In addition, $\mathrm{Ba}(\mathrm{Fe}, \mathrm{Co})_{2} \mathrm{As}_{2}$ is relatively stable and many references on single crystals and films are available for comparison. Phase formation, microstructure, magnetic and transport properties of $\mathrm{Ba}(\mathrm{Fe}, \mathrm{Co})_{2} \mathrm{As}_{2}$ polycrystalline bulks prepared by systematically varied starting materials, mixing and heat treatment conditions are comparatively studied.

\section{Experimental}

All the powder processing was performed under high purity Ar atmosphere using a glove box to avoid oxygen contamination. Cobalt doped Ba122 polycrystalline bulk samples were synthesized by three different processes (hereafter, denoted as Process A, Process B and Process C) using different starting powders. For Process A, starting materials of $\mathrm{Ba}$, FeAs and $\mathrm{CoAs}$ powders were weighed in stoichiometric ratio to form $\mathrm{Ba}\left(\mathrm{Fe}_{0.92} \mathrm{Co}_{0.08}\right)_{2} \mathrm{As}_{2}$, mixed in an agate mortar. Then, the mixed powder was pressed into a pellet, sealed in an evacuated quartz ampoule, and heated at $900^{\circ} \mathrm{C}$ for $24-240 \mathrm{~h}$. For Process B, Ba, FeAs and CoAs powders were mixed and ground by high-energy ball-milling (HEBM). For Process $\mathrm{C}, \mathrm{Ba}\left(\mathrm{Fe}_{0.92} \mathrm{Co}_{0.08}\right)_{2} \mathrm{As}_{2}$ bulks were synthesized from elemental metals, i.e. $\mathrm{Ba}, \mathrm{Fe}$, $\mathrm{Co}$ and As, which were mixed and ground by HEBM. The so-obtained starting powders were pressed and heated by the similar processes with the Process A. The heat treatment conditions for the samples shown in Figs. 1-4 and 5(a) are $900^{\circ} \mathrm{C}$ for $48 \mathrm{~h}$ with resintering at $900^{\circ} \mathrm{C}$ for $24 \mathrm{~h}, 900^{\circ} \mathrm{C}$ for $48 \mathrm{~h}$, and $600^{\circ} \mathrm{C}$ for $48 \mathrm{~h}$ for the Processes $\mathrm{A}, \mathrm{B}$ and $\mathrm{C}$, respectively.

The constituent phases of the samples were analyzed by powder X-ray diffraction (XRD) using Cu- $K_{\alpha}$ radiation. Microstructural observations were performed on the cross-sectional polished surface by scanning electron microscopy (Hitachi High-Technologies: S-3400N; JEOL: JSM-7001F). Transport measurements were performed by the conventional four-point probe method with a $16 \mathrm{~Hz}$ AC current using a physical property measurement system (Quantum Design: PPMS Model 6000). Magnetization was measured using a SQUID magnetometer (Quantum Design: MPMS-XL5s). 

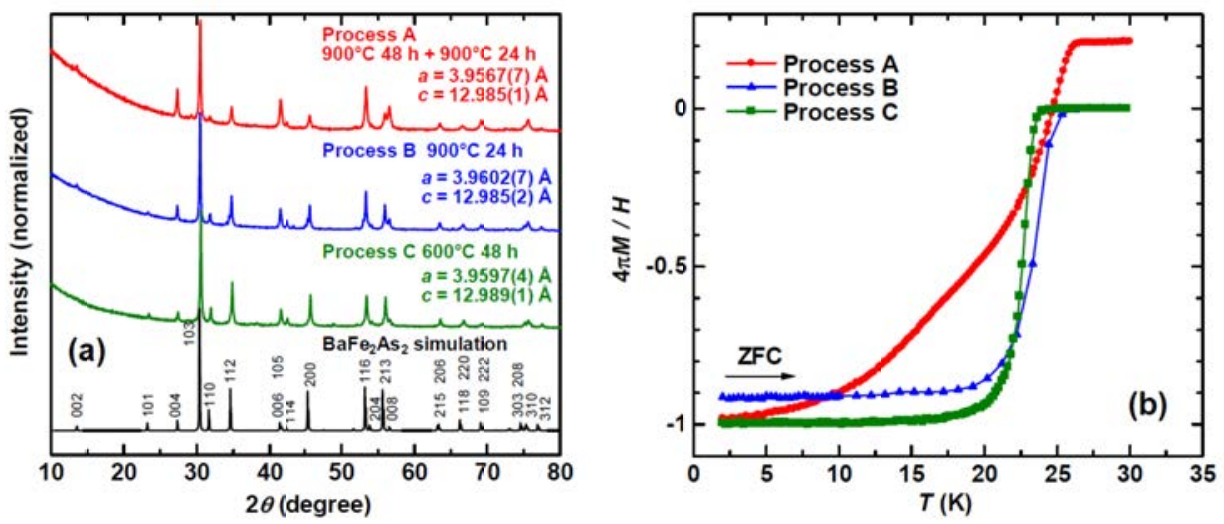

Fig. 1. (a) Powder $\mathrm{XRD}$ patterns for $\mathrm{Ba}\left(\mathrm{Fe}_{0.92} \mathrm{Co}_{0.08}\right)_{2} \mathrm{As}_{2}$ polycrystalline bulks prepared by the three different processes $\mathrm{A}, \mathrm{B}$ and $\mathrm{C}$ (from top to bottom). Simulation pattern for $\mathrm{BaFe}_{2} \mathrm{As}_{2}(a=3.987 \AA, c=13.001 \AA)$ obtained by Rietveld analysis is shown for reference. (b) Temperature dependence of magnetization under 1 Oe for zero-field-cooled $\mathrm{Ba}\left(\mathrm{Fe}_{0.92} \mathrm{Co}_{0.08}\right)_{2} \mathrm{As}_{2}$ polycrystalline bulks.
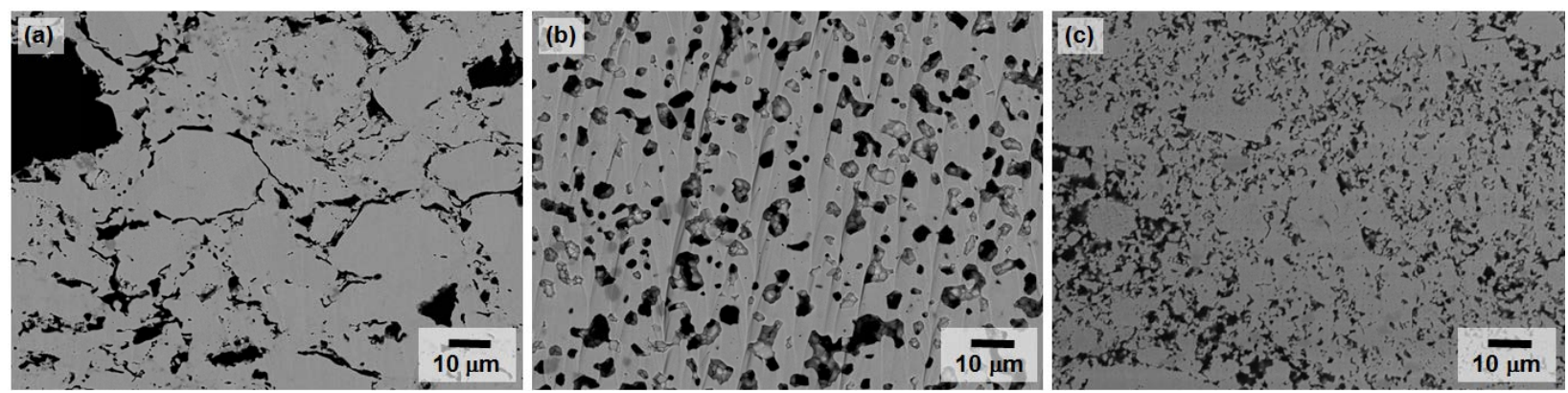

Fig. 2. Backscattered electron images for the cross-sectional polished surface of the $\mathrm{Ba}\left(\mathrm{Fe}_{0.92} \mathrm{Co}_{0.08}\right)_{2} \mathrm{As}_{2}$ polycrystalline bulks prepared by Process A (a), Process B (b), and Process C (c).

\section{Results and discussions}

In order to synthesize $\mathrm{Ba}(\mathrm{Fe}, \mathrm{Co})_{2} \mathrm{As}_{2}$ phase with high reproducibility, firstly heat treatment condition was optimized for the Process A. By heating at a temperature of above $900^{\circ} \mathrm{C}, \mathrm{Ba}(\mathrm{Fe}, \mathrm{Co})_{2} \mathrm{As}_{2}$ was successfully obtained as main phase. The phase purity was further improved by introducing regrinding and resintering process at $900^{\circ} \mathrm{C}$ for $24 \mathrm{~h}$. Powder XRD pattern for the resintered sample (Fig. 1(a)) shows that the sample is almost single phase $\mathrm{Ba}(\mathrm{Fe}, \mathrm{Co})_{2} \mathrm{As}_{2}$. Figure 1(b) shows the temperature dependence of magnetization for the samples synthesized by the different processes. The sample prepared by the Process A showed high $T_{\mathrm{c}}{ }^{\text {onset }}$ over $25 \mathrm{~K}$. However, superconducting transition is broad and magnetic background can be seen. These results suggest weak bulk superconducting current and existence of ferromagnetic impurities, such as unreacted FeAs and CoAs, respectively. To reduce such unreacted phases by enhancing reactivity, grinding raw materials into fine powder is expected to be effective. The sample synthesized by the Process B using HEBM powder shows no magnetic background and high $T_{\mathrm{c}}$ onset equivalent to the sample by the Process A (Fig. 1(b)). Moreover superconducting transition becomes sharper indicating the existence of bulk shielding current owing to much improved intergranular electromagnetic coupling.

Figures 2 (a) and (b) show backscattered electron images for the cross-sectional polished surface of the samples synthesized by the Processes A and B, respectively. In these images, the light gray matrix represents 122 phase, the dark gray region represents $(\mathrm{Fe}, \mathrm{Co}) \mathrm{As}$ phase and black region represents void. In the sample prepared by the Process A, large voids interrupted physical contact between grains with several to several tens micrometers in size. In addition, unreacted $(\mathrm{Fe}, \mathrm{Co})$ As phase with several micrometers in size was observed. On the other hand, in the sample prepared by the Process $\mathrm{B}$, strongly connected $\mathrm{Ba}(\mathrm{Fe}, \mathrm{Co})_{2} \mathrm{As}_{2}$ matrix which is composed of fine grains with 2-3 micrometers in size and homogeneously distributed small voids can be observed. The size and number of impurity $(\mathrm{Fe}, \mathrm{Co})$ As region were reduced. 

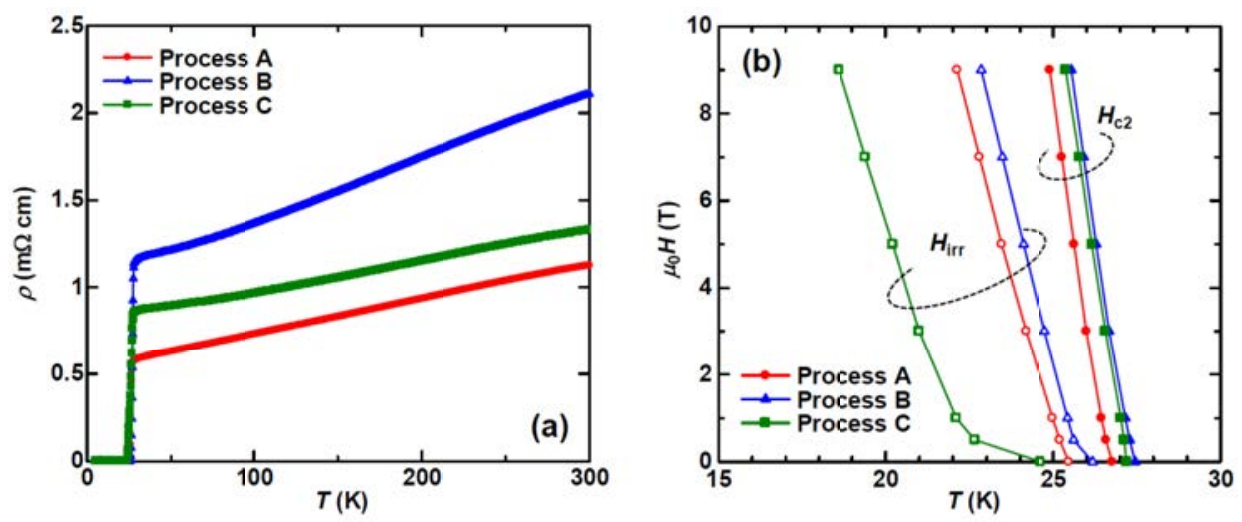

Fig. 3. Transport properties of $\mathrm{Ba}\left(\mathrm{Fe}_{0.92} \mathrm{Co}_{0.08}\right)_{2} \mathrm{As}_{2}$ polycrystalline bulks prepared by the three different processes A, B and C; (a) Temperature dependence of resistivity under zero external field. (b) Temperature dependence of upper critical field $\left(H_{\mathrm{c} 2}\right)$ and irreversibility field $\left(H_{\mathrm{irr}}\right)$.

By applying HEBM in the Process $\mathrm{B}$, we confirmed that $\mathrm{Ba}(\mathrm{Fe}, \mathrm{Co})_{2} \mathrm{As}_{2}$ can be synthesized as main phase at lower temperature $\sim 600^{\circ} \mathrm{C}$, which is below the sublimation point of arsenic $\left(615^{\circ} \mathrm{C}\right)$. Then elemental metal arsenic was used instead of FeAs and CoAs as an arsenic source (the Process C). Like the Process B, the sample synthesized at $600^{\circ} \mathrm{C}$ by the Process $\mathrm{C}$ is nearly single phase $\mathrm{Ba}(\mathrm{Fe}, \mathrm{Co})_{2} \mathrm{As}_{2}$ (Fig. 1(a)) and shows sharp transition without magnetic background (Fig. 1(b)). Backscattered electron image of the sample prepared by the Process $\mathrm{C}$ shows that the sizes of both $\mathrm{Ba}(\mathrm{Fe}, \mathrm{Co})_{2} \mathrm{As}_{2}$ grains and voids are further refined and rather uniformly distributed (Fig. 2(c)).

Figure 3(a) shows temperature dependence of resistivity under zero external field. All the samples show metallic temperature dependence of resistivity and sharp superconducting transition with high $T_{\mathrm{c}}$ onset over $27 \mathrm{~K}$. The resistivity values at room temperature for the samples prepared by the Processes A, B and C are 1.1, 2.1 and 1.3 $\mathrm{m} \Omega \mathrm{cm}$, respectively. The samples showed high $T_{\mathrm{c}}$ and low resistivity suggesting that the samples are optimally doped and with high phase purity. Figure 3(b) shows temperature dependence of $H_{\mathrm{c} 2}$ and irreversibility field $\left(H_{\mathrm{irr}}\right)$ defined by $90 \%$ and $10 \%$, respectively, of the in-field resistive transitions. Note that this definition of $H_{\text {irr }}$ does not strictly correspond to $T_{R=0}$. All the samples showed steep $H_{\mathrm{c} 2}$ slope $d H_{\mathrm{c} 2} / d T(-5.0,-4.9$ and $-4.8 \mathrm{~T} / \mathrm{K}$ for the samples prepared by the Processes A, B and C, respectively), which is comparable to $d H_{\mathrm{c} 2}{ }^{a b} / d T=-4.9 \mathrm{~T} / \mathrm{K}$ of single crystal [7]. The samples prepared by the Process A and B show small $\Delta T_{\mathrm{c}}=T_{\mathrm{c} 2}-T_{\mathrm{irr}} \sim 1.3 \mathrm{~K}$ and $\Delta T_{\mathrm{c}}$ under external field too maintained rather small as shown in Fig. 3(b). On the other hand, the sample prepared by the Process $\mathrm{C}$ showed strong upturn of $H_{\text {irr }}(T)$ while the slope of $d H_{\text {irr }} / d T=-2.5 \mathrm{~T} / \mathrm{K}$ under high field is comparable to -2.8 and $-3.1 \mathrm{~T} / \mathrm{K}$ of the samples prepared by the Processes A and B, respectively. The sensitivity of $T_{\text {irr }}$ to relatively weak external field $\sim 0.5 \mathrm{~T}$ suggests that grain boundaries of Ba122 is electromagnetically weakly coupled and may work as Josephson junction due to thin normal wetting phase and/or intrinsic weak-link, as was observed in Sm1111 [21].

Figure 4(a) shows magnetic hysteresis loops at $5 \mathrm{~K}$. The sample prepared by the Process A showed ferromagnetic background, which is in good agreement with the susceptibility measurement and microstructural analysis. Figure 4(b) shows the magnetic field dependence of $J_{\mathrm{c}}$ at $5 \mathrm{~K}$ derived from the hysteresis loop width $\Delta M$ using the extended Bean model $J_{\mathrm{c}}=20 \Delta M / a(1-a / 3 b)$. It should be noted that the estimated $J_{\mathrm{c}}$ by this Bean model is affected by contributions from both inter- and intra-granular currents and thus overestimated for granular superconducting polycrystals. This expression yields a $J_{\mathrm{c}}$ ranging from 1,000 to $5,000 \mathrm{~A} / \mathrm{cm}^{2}$ under self-field. $J_{\mathrm{c}}$ shows rapid decrease under low field below $1 \mathrm{~T}$ and then becomes rather independent of field up to $5 \mathrm{~T}$. Larger hysteresis loop width and mean $J_{\mathrm{c}}$ for the sample prepared by the Process A can be attributed to intra-granular currents because of the large grain size as was observed in Fig. 2(a).

In order to quantitatively evaluate the inter-granular $J_{\mathrm{c}}$ of the bulk samples, remanent magnetization analysis was performed. The bulk samples were exposed to many cycles of ever increasing magnetic field $H_{\mathrm{a}}$, followed by removal of the field and measurement of the remanent magnetization $\left(M_{\mathrm{R}}\right)$ [16]. Figure 5(a) shows derivative of $M_{\mathrm{R}}$ $\left(d M_{\mathrm{R}} / d H_{\mathrm{a}}\right)$ as a function of maximum applied field $H_{\mathrm{a}}$. Like Sm1111 bulks [16, 29], the sample synthesized by the Process A shows two clearly separated peaks due to two different scales of current flow, i.e. intergranular and locally circulating current. The lower field peak (indicated by arrows) which has its origin in $J_{\mathrm{c}}{ }^{\text {global }}$ shifted more than one order of magnitude towards higher field for the samples synthesized by the Processes B and C, suggesting 

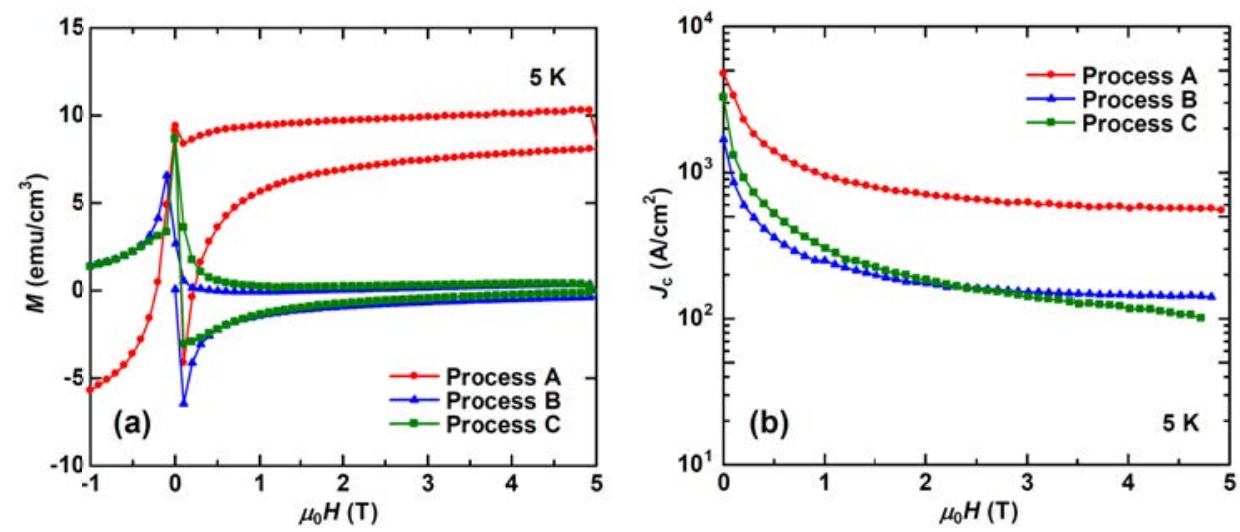

Fig. 4. Magnetic properties of $\mathrm{Ba}\left(\mathrm{Fe}_{0.92} \mathrm{Co}_{0.08}\right)_{2} \mathrm{As}_{2}$ polycrystalline bulks prepared by the three different processes $\mathrm{A}, \mathrm{B}$ and $\mathrm{C}$; (a) Magnetic hysteresis loop at $5 \mathrm{~K}$. (b) Magnetic field dependence of $J_{\mathrm{c}}$ at $5 \mathrm{~K}$ calculated by the extended Bean model.
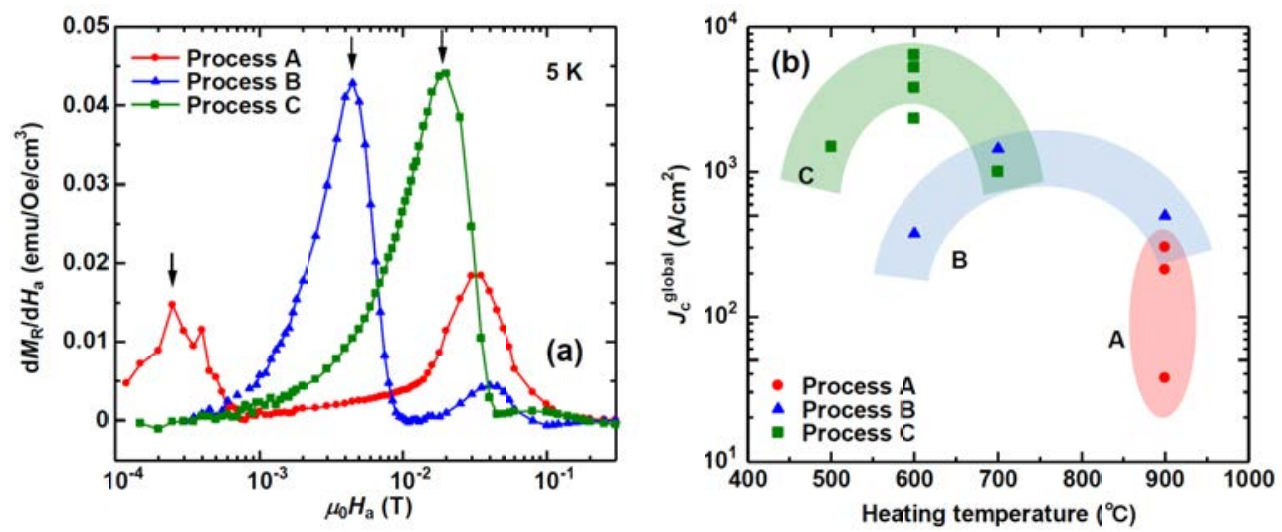

Fig. 5. (a) Maximum applied field dependence of the derivatives of remanent magnetization for the $\mathrm{Ba}\left(\mathrm{Fe}_{0.92} \mathrm{Co}_{0.08}\right)_{2} \mathrm{As}_{2}$ polycrystalline bulks prepared by the three different processes $\mathrm{A}, \mathrm{B}$ and $\mathrm{C}$. The lower field peaks due to intergranular current magnetization are indicated by arrows. (b)

The relationship between heating temperature and intergranular critical current density $J_{\mathrm{c}}{ }^{\text {global }}$ at $5 \mathrm{~K}$ for $\mathrm{Ba}\left(\mathrm{Fe}_{0.92} \mathrm{Co}_{0.08}\right)_{2} \mathrm{As}_{2}$ bulks.

that intergranular connection was much improved in these samples. Assuming that current is flowing over the whole sample, the Bean model shows that the inter-granular $J_{\mathrm{c}}$ is given by $J_{\mathrm{c}}^{\text {global }}=2 \mathrm{H}_{\text {peak } 1} / \mathrm{w}$ where $H_{\text {peak } 1}$ is lower field peak in $d M_{\mathrm{R}} / d H_{\mathrm{a}}\left(H_{\mathrm{a}}\right)$ (Fig. 5(a)) and $w$ is the sample size which is typically $\sim 1 \mathrm{~mm}$. The estimated $J_{\mathrm{c}}{ }^{\text {global }}$ for the samples prepared by the Process A (heated at $900^{\circ} \mathrm{C}$ for $48 \mathrm{~h}$ and resintered at $900^{\circ} \mathrm{C}$ for $24 \mathrm{~h}$ ), the Process $\mathrm{B}$ (heated at $900^{\circ} \mathrm{C}$ for $48 \mathrm{~h}$ ) and the Process $\mathrm{C}$ (heated at $600^{\circ} \mathrm{C}$ for $48 \mathrm{~h}$ ) are 40,500 , and 2,300 A/cm ${ }^{2}$, respectively.

Finally the relationship between heating temperature and $J_{\mathrm{c}}{ }^{\text {global }}$ for samples prepared by the three processes is summarized in Fig. 5(b). It can be clearly seen that $J_{c}{ }^{\text {global }}$ improved with decreasing the reaction temperature by using the activated HEBM powders for the Processes B and C. With increasing the heating duration at $600^{\circ} \mathrm{C}, J_{\mathrm{c}}$ global progressively improved and high $J_{\mathrm{c}}{ }^{\text {global }}$ of $6,000 \mathrm{~A} / \mathrm{cm}^{2}$ which is the highest as for polycrystalline $\mathrm{Ba}(\mathrm{Fe}, \mathrm{Co})_{2} \mathrm{As} s_{2}$ samples prepared under ambient pressure was obtained for the sample heated at $600^{\circ} \mathrm{C}$ for $120 \mathrm{~h}$ by the Process $\mathrm{C}$.

Here we briefly discuss the factors affecting $J_{c}{ }^{\text {global }}$. TEM analyses [30] (not shown here) showed that wetting phase exists at grain boundaries in the samples prepared by the Processes A and $\mathrm{C}$ while grain boundaries of the sample prepared by the Process B is clean. At the present stage we do not fully understand the origin of large difference in $J_{\mathrm{c}}$ global values among the different processes. For the sample prepared by the Process A, poor phase purity, wetting phases and large pores which separate connection between grains/particles can be addressed for the limiting factors for $J_{\mathrm{c}}{ }^{\text {global }}$. From microstructural view point, we expect that the sample prepared by the Process B at $900^{\circ} \mathrm{C}$ with better phase purity and well-connected grain boundaries would show the highest $J_{\mathrm{c}}^{\text {global }}$. That the samples prepared by the Process C, especially synthesized at lower temperatures, showed higher $J_{\mathrm{c}}{ }^{\text {global }}$ suggests that fine grain size and grain boundary structure formed at low temperature are favorable for quantitatively increasing the area of effective transport current path, while they are qualitatively weak and suppressed under low external field of $\sim 0.5 \mathrm{~T}$. Detailed microstructural analyses on grain boundaries should be examined to clarify the mechanism. 


\section{Summary}

Polycrystalline $\mathrm{Ba}(\mathrm{Fe}, \mathrm{Co})_{2} \mathrm{As}_{2}$ bulk samples were prepared by systematically varied processes to find the effective routes for high intergranular critical current $J_{c}^{\text {global }}$. Activated powder prepared by high-energy ball-milling process yielded homogeneous and well-connected microstructure with fine grain size and better phase purity even by heating at lower temperature. As a consequence relatively high $J_{\mathrm{c}}^{\text {global }}$ exceeding $6,000 \mathrm{~A} / \mathrm{cm}^{2}$ was achieved for the sample heated at $600^{\circ} \mathrm{C}$ for $120 \mathrm{~h}$. On the other hand, magnetic and transport measurement showed rapid deterioration of intergranular current under external field, suggesting that the grain boundaries are weakly coupled.

\section{Acknowledgements}

The authors gratefully acknowledge R. Murai, S. Tsujitake, S. Ohtsuka and T. Moroyama (The University of Tokyo) for experimental assistance. This work was supported by JST PRESTO and SICORP. Microstructural analysis was conducted at the Center for Nano Lithography \& Analysis, University of Tokyo, supported by MEXT.

\section{References}

[1] Kamihara $Y$ et al. Iron-based layered superconductor $\mathrm{La}\left[\mathrm{O}_{1-x} \mathrm{~F}_{x}\right] \mathrm{FeAs}(x=0.05-0.12)$ with $T_{\mathrm{c}}=26 \mathrm{~K}$. J Am Chem Soc 2008;130:3296-7.

[2] Rotter M, Tegel M, Johrendt D. Superconductivity at $38 \mathrm{~K}$ in the Iron Arsenide $\left(\mathrm{Ba}_{1-x} \mathrm{~K}_{x}\right) \mathrm{Fe}_{2} \mathrm{As}_{2}$. Phys Rev Lett 2008;101:107006 1-4.

[3] Sefat AS et al. Superconductivity at $22 \mathrm{~K}$ in Co-doped $\mathrm{BaFe}_{2} \mathrm{As}_{2}$ crystals. Phys Rev Lett 2008;101:117004 1-4.

[4] Hsu FC et al. Superconductivity in the PbO-type structure $\alpha$-FeSe. Proc Natl Acad Sci USA 2008 ; 105 : 14262-4.

[5] Jaroszynski J et al. Upper critical fields and thermally-activated transport of $\mathrm{NdFeAsO}_{0.7} \mathrm{~F}_{0.3}$ single crystal. Phys Rev B 2008; 78:174523 1-9.

[6] Yuan HQ et al. Nearly isotropic superconductivity in (Ba,K)Fe ${ }_{2} \mathrm{As}_{2}$. Nature 2008;457:565-8.

[7] Yamamoto A et al. Small anisotropy, weak thermal fluctuations, and high field superconductivity in Co-doped iron pnictide $\mathrm{Ba}\left(\mathrm{Fe}_{1-x} \mathrm{Co}_{x}\right)_{2} \mathrm{As}_{2}$. Appl Phys Lett 2009;94: 062511 1-3.

[8] Yang $\mathrm{H}$ et al. Fishtail effect and the vortex phase diagram of single crystal $\mathrm{Ba}_{0.6} \mathrm{~K}_{0.4} \mathrm{Fe}_{2} \mathrm{As}_{2}$. Appl Phys Lett 2008;93:142506 1-3.

[9] Moll PJW et al. High magnetic-field scales and critical currents in $\operatorname{SmFeAs}(\mathrm{O}, \mathrm{F})$ crystals. Nat Mater 2010;9:628-633.

[10] Katase T, Hiramatsu H, Kamiya T, Hosono H. High critical current density $4 \mathrm{MA} / \mathrm{cm}^{2}$ in Co-doped $\mathrm{BaFe}_{2} \mathrm{As}_{2}$ epitaxial films grown on $(\mathrm{La}, \mathrm{Sr})(\mathrm{Al}, \mathrm{Ta}) \mathrm{O}_{3}$ substrates without buffer layers. Appl Phys Express 2010;3:063101 1-3.

[11] Lee $\mathrm{S}$ et al. Template engineering of Co-doped $\mathrm{BaFe}_{2} \mathrm{As}_{2}$ single-crystal thin films. Nat Mater 2010;9:397-402.

[12] Iida $\mathrm{K}$ et al. Epitaxial Growth of Superconducting $\mathrm{Ba}\left(\mathrm{Fe}_{1-x} \mathrm{Co}_{x}\right)_{2} \mathrm{As}_{2}$ Thin films on technical Ion Beam Assisted Deposition MgO substrates. Appl Phys Express 2011;4:013103 1-3.

[13] Ueda S, Takeda S, Takano S, Yamamoto A, Naito M. High- $T_{\mathrm{c}}$ and high- $J_{\mathrm{c}} \operatorname{SmFeAs}(\mathrm{O}, \mathrm{F})$ films on fluoride substrates grown by molecular beam epitaxy. Appl Phys Lett 2011;99:232505 1-3.

[14] Sakagami A et al. Critical current density and grain boundary property of $\mathrm{BaFe}_{2}(\mathrm{As}, \mathrm{P})_{2}$ thin films. Physica C 2013;494:181-184.

[15] Putti M et al. New Fe-based superconductors: properties relevant for applications. Supercond Sci Technol 2010;23: 034003 1-10.

[16] Yamamoto A et al. Evidence for two distinct scales of current flow in polycrystalline Sm and Nd iron oxypnictides. Supercond Sci Technol 2008;21:095008 1-8.

[17] Polichetti $\mathrm{M}$ et al. Granularity and vortex dynamics in $\mathrm{LaFeAsO}_{0.92} \mathrm{~F}_{0.08}$ probed by harmonics of the ac magnetic susceptibility. Phys Rev B $2008 ; 78: 2245231-10$.

[18] Tamegai T et al. Bulk and local magnetic properties of iron-based oxypnictide $\mathrm{SmFeAsO}_{1-x} \mathrm{~F}_{x}$. J Phys Soc Jpn 2008;77:C 54-57.

[19] Lee $\mathrm{S}$ et al. Weak-link behavior of grain boundaries in superconducting $\mathrm{Ba}\left(\mathrm{Fe}_{1-x} \mathrm{Co}_{x}\right)_{2} \mathrm{As}_{2}$ bicrystals. Appl Phys Lett 2009;95:212505 1-3.

[20] Katase T et al. Advantageous grain boundaries in iron pnictide superconductors. Nat Commun 2011;2:409 1-6.

[21] Kametani F et al. Intergrain current flow in a randomly oriented polycrystalline SmFeAsO $\mathrm{A}_{0.85}$. Appl Phys Lett 2009;95:142502 1-3.

[22] Durrell JH et al. The behavior of grain boundaries in the Fe-based superconductors. Rep Prog Phys 2011;74:124511 1-23.

[23] Weiss JD et al. High intergrain critical current density in fine-grain $\left(\mathrm{Ba}_{0.6} \mathrm{~K}_{0.4}\right) \mathrm{Fe}_{2} \mathrm{As}_{2}$ wires and bulks. Nat Mater 2012;11:682-5.

[24] Togano K, Gao Z, Taira H, Ishida S, Kihou K, Iyo A, Eisaki H, Matsumoto A, Kumakura H. Enhanced high-field transport critical current densities observed for ex situ PIT processed Ag/(Ba, K)Fe ${ }_{2} \mathrm{As}_{2}$ thin tapes. Supercond Sci Technol 2013;26:065003 1-9.

[25] Yao C, Lin H, Zhang X, Wang D, Zhang Q, Ma Y, Awaji S, Watanabe K. Microstructure and transport critical current in $\mathrm{Sr}_{0.6} \mathrm{~K}_{0.4} \mathrm{Fe}_{2} \mathrm{As}_{2}$ superconducting tapes prepared by coldpressing. Supercond Sci Technol 2013;26:075003 1-7.

[26] Wang L, Qi Y, Wang D, Gao Z, Zhang X, Zhang Z, Wang C, Ma Y. Low-temperature synthesis of $\mathrm{SmFeAsO}_{0.7} \mathrm{~F}_{0.3-\delta}$ wires with a high transport critical current density. Supercond Sci Technol 2010;23:075005 1-8.

[27] Fujioka M, Kota T, Matoba M, Ozaki T, Takano Y, Kumakura H, Kamihara Y. Effective ex-situ fabrication of F-doped SmFeAsO wire for high transport critical current density. Appl Phys Express 2011;4:063102 1-3.

[28] Ozaki T et al. Fabrication of binary FeSe superconducting wires by diffusion process. J Appl Phys 2012;111:112620 1-3.

[29] Eisterer M et al. Disorder effects and current percolation in FeAs-based superconductors. Supercond Sci Technol 2010;23:054006 1-7.

[30] Shimada Y et al. in preparation. 